this

phenomenon

of complex

rearrangements

was termed 'chromoplexy'
$\Rightarrow$

\section{CANCER GENOMICS}

\section{Coming in waves}

One benefit of large-scale cancer genome-sequencing projects is that they are poised to give us insights into temporal alterations in cancer genomes and thus into how these alterations contribute to tumour initiation and progression. In order to better understand the genomic architecture and evolution of prostate cancer, Levi Garraway, Francesca Demichelis and colleagues conducted whole-genome sequencing of 57 prostate tumours and matched normal tissue, and they used computer modelling to analyse how genomic rearrangements arise.

The authors found that genomic rearrangements in these samples often occurred in the context of a 'chain' of events, and systematic analysis of the chains indicated that most of these lead to DNA deletions and involve the fusion of breakpoints at either end of the deletion to novel partners (rather than to each other). The tumour data were compared with a model of the probability that pairs of neighbouring DNA breakpoints would arise independently of each other; this analysis revealed a deviation between the two, suggesting that these rearrangements are likely to be interdependent. To further understand how the chains arise, the authors created an algorithm called ChainFinder, and this revealed that several chains often occurred within the same tumour (63\% of tumours had two or more chains), and that chains had variable numbers of rearrangements ranging from three to more than 40 . This phenomenon of complex rearrangements was termed 'chromoplexy' (derived from the Greek word pleko, which means 'to weave' or 'to braid').
Looking at specific subsets of prostate tumours, the authors found differences in chromoplexy. The tumours that had oncogenic fusions containing the ETS family of transcription factors had more interchromosomal rearrangements and single events involving more than one chromosome compared with ETS ${ }^{-}$ tumours; given that ETS fusions have been shown to arise during androgen receptor (AR)-mediated transcription, the authors hypothesized that chromoplexy in this context might be coupled to transcriptional processes. Interestingly, the pattern of rearrangement seen in ETS ${ }^{-}$tumours was more reminiscent of chromothripsis and might arise following the loss of the putative tumour suppressor gene encoding chromodomain helicase DNA binding protein 1 (CHD1).

Overall, chromoplexy frequently led to simultaneous deletion or rearrangement of common cancer genes, and to try to understand this further the authors examined the clonal status of each change. Several common deletions - including NK3 homeobox 1 (NKX3-1) and the deletion that produces the ETS fusion TMPRSS2-ERG - were clonal, whereas others, such as deletion of PTEN, were subclonal. On the basis of this, the authors proposed a 'consensus path' of progression for a typical prostate tumour involving these and other common lesions. Interestingly, the presence of rearrangement chains in both clones and subclones indicates that multiple rounds of chromoplexy may occur, leading the authors to propose that the evolution of these tumours is punctuated.

These data give us a clearer picture of the genomic architecture and history of prostate tumours and provide clues to the chronology of oncogenic events. However, it is important to note that the concept of chromoplexy is derived from computational modelling, and mechanisms by which it may occur need to be confirmed experimentally.

Sarah Seton-Rogers

ORIGINAL RESEARCH PAPER Baca, S. C. et al.

Punctuated evolution of prostate cancer genomes. Cell 153, 666-677 (2013)

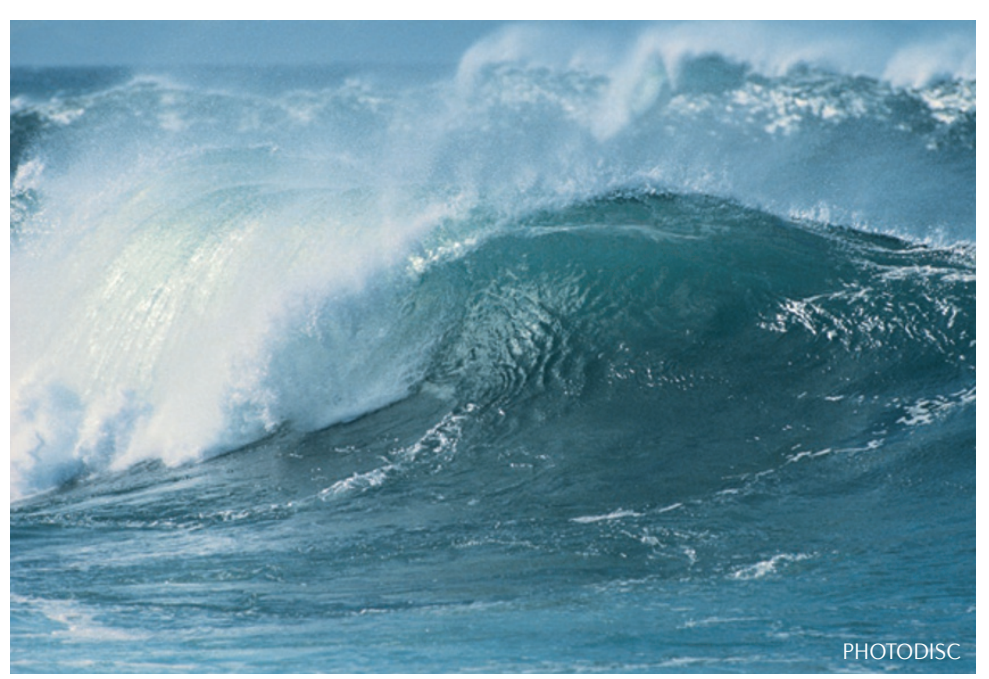

